\title{
A Study on Effective Elastic Modulus of Nanocomposites
}

\author{
Li-Chun BIAN ${ }^{1, a,{ }^{*},}$ Jing PAN ${ }^{1}$ and Cai-Hua WANG ${ }^{1}$ \\ ${ }^{1}$ Key Laboratory of Mechanical Reliability for Heavy Equipments and Large Structures \\ of Hebei Province, Yanshan University, Qinhuangdao 066004, PR China \\ ae-mail address: bianlic@126.com
}

${ }^{*}$ Corresponding author

Keywords: Elastic modulus, Carbon nanotube, Nanocomposite, Fiber, Waviness.

\begin{abstract}
Nanocomposites reinforced by carbon nanotubes exhibit great advantages in engineering applications. The waviness of nanotubes is one of the important factors which affect the effective elastic modulus of nanocmposites. This paper aims to develop a new model to replace the wavy carbon nanotubes with effective fiber. The effective modulus of nanocomposites with randomly oriented fibers can be predicted based on the micromechanics. The effects of waviness and agglomeration on the effective modulus are also analyzed. It is shown that they can reduce the stiffness of carbon nanotubes, significantly. Moreover, the effective elastic modulus of nanocomposites is very sensitive to the waviness and the agglomeration.
\end{abstract}

\section{Introduction}

To-date, many researchers have studied on the effective elastic modulus of nanocomposites. From the discovery of carbon nanotube (CNT), its remarkable properties attract much attention of scholars [1-3]. Many effects, e.g., CNT geometry and dispersion, ratio of length and diameter, and interfacial bonding on mechanical properties of CNT-reinforced composites have been investigated in some ways [4-6]. Shady and Gowayed [7] investigated the effect of waviness on effective modulus of composite based on the fact assuming the CNT as a special helical shape. It has been found that the effect of curvature seems more critical with increaseing weight fraction. Moreover, the strain energy of nanotube and the effective fiber are predicted, and the constraints imposed by the matrix on the deformation are considered in this study.

As compared with an experimental analysis, numerical method is often employed as an effective way for the mechanical behavior of nanocomposites, since it cost less and doesn't need to establish complex evaluation. Giannopoulos et al. [8] gives a micromechanical finite element approach for the estimation of effective Young's modulus. In the work of Giannopoulos et al. [8], a cylindrical representative, which consists of reinforcement, interface and matrix material is employed, and a discrete spring-based finite element method is used to simulate the nanotubes. Based on the finite element method, it has been found that an increase in the amount of randomness results in decreased Young's modulus values and strength of composite [9].

The aim of present paper is to investigate the effective elastic modulus of CNT-reinforced composites. We will pay more attention to the influence of CNT waviness and agglomeration on the composite. A spatial wavy fiber with a solid circular cross-section is used to simulate the actual CNT state in the matrix. Then, we replace the spatial wavy fiber with a straight fiber along the axis and a coin shape wafer. Both fiber and coin shape are assumed to be isotropic, and then the effective modulus can be determined by the Mori-Tanaka effective-field method propose.

\section{Micromechanics Model}

Many experimental results have shown that CNTs generally exist in a wavy shape in the nanocomposites. In this study, paramters $a$ and $\lambda$ are the amplitude and wavelength of spatial wavy fiber. The diameter and volume fraction of CNTs are denoted by $d$ and $f_{\mathrm{r}}$. Then, we replace the 
spatial wavy fiber with a straight fiber along the axis and a coin shape wafer. The length and the volume fraction of straight fiber are denoted by $\lambda$ and $f_{1}$. The radius, the volume fraction, and the thickness of wafers are denoted by $a, f_{2}$ and $h$, respectively.

Considering a representative volume $V$ of a CNT-reinforced composite, we have the relations:

$$
\begin{aligned}
& f_{1}=N \frac{\pi d^{2} \lambda}{4 V} \\
& f_{2}=N \frac{\pi a^{2} h}{4 V} \\
& h=\frac{d^{2}}{a}
\end{aligned}
$$

where $N$ is the number of a certain CNTs with same waviness which is identified by the radio a/ $\lambda$ denoted by $\delta$. From Eqs. 1, 2, and 3, we can obtain:

$$
\frac{f_{1}}{f_{2}}=\frac{\lambda}{a}
$$

In order to remain the volume fraction, it is assumed that the sum of straight fiber volume and wafer volume equals to the carbon nanotube volumes. Then, we can express this relation as,

$$
f_{1}+f_{2}=f_{r}
$$

Summarizing equations above, we can obtain:

$$
\begin{aligned}
& f_{1}=\frac{f_{r}}{1+\delta} \\
& f_{2}=\frac{\delta \cdot f_{r}}{1+\delta}
\end{aligned}
$$

It is assumed that the spatial CNT horizontally effective modulus is provided by the straight fiber. Through the rules-of -mixtures, we get

$$
\begin{aligned}
& f_{1} E_{1}+f_{m} E_{m}=E_{x} \\
& f_{2} E_{2}+f_{m} E_{m}=E_{y}
\end{aligned}
$$

where $E_{\mathrm{m}}$ is the Young's modulus of matrix, $f_{\mathrm{m}}$ and $f_{1}$ are the volume fractions of matrix and straight fibers, respectively. Here, we adopt the result of effective longitudinal modulus [10],

$$
E_{x}=\frac{(1+\mathrm{c})^{3 / 2}}{\left(1+\frac{\mathrm{c}}{2}\right) \cdot S_{11}-\left[1+\frac{3 \mathrm{c}}{2}-(1+\mathrm{c})^{3 / 2}\right] \cdot \mathrm{S}_{22}+\frac{c}{2}\left(2 \mathrm{~S}_{12}+\mathrm{S}_{66}\right)}
$$




$$
E_{y}=\frac{(1+\mathrm{c})^{3 / 2}}{\left(1+\frac{\mathrm{c}}{2}\right) \cdot \mathrm{S}_{22}+\left[(1+\mathrm{c})^{3 / 2}-1-\frac{3 \mathrm{c}}{2}\right] \cdot \mathrm{S}_{11}+\frac{\mathrm{c}}{2} \cdot\left(2 \mathrm{~S}_{12}+\mathrm{S}_{66}\right)}
$$

where

$$
\begin{aligned}
& C=\left(\frac{2 \pi \mathrm{a}}{\lambda}\right)^{2}=4 \pi^{2} \delta^{2} \\
& S_{11}=\frac{1}{E_{L}} ; S_{22}=\frac{1}{E_{T}} \\
& S_{12}=-\frac{v_{L T}}{E_{L}} ; S_{66}=\frac{1}{G_{L T}}
\end{aligned}
$$

In the above formulas, $E_{\mathrm{L}}, E_{\mathrm{T}}$, and $G_{\mathrm{LT}}$ are the longitudinal, transverse and shear moduli of composite with wavy fibers. Term $v_{\mathrm{LT}}$ is the longitudinal-transverse Poisson's ratio. The rules-of -the mixtures can be used to obtain these parameters,

$$
\begin{aligned}
& E_{L}=f_{r} E_{r}+f_{m} E_{m} \\
& E_{T}=\left(\frac{f_{r}}{E_{r}}+\frac{f_{m}}{E_{m}}\right)^{-1} \\
& v_{L T}=f_{r} v_{r}+f_{m} v_{m}
\end{aligned}
$$

According the intrinsic relationship, we can get:

$$
G_{f}=\frac{E_{f}}{2\left(1+v_{f}\right)}, G_{m}=\frac{E_{m}}{2\left(1+v_{\mathrm{m}}\right)}
$$

From the above analysis we can get the effective elastic modulus of straight and wafer,

$$
\begin{aligned}
& E_{1}=\frac{E_{x}-f_{m} E_{m}}{f_{1}} \\
& E_{2}=\frac{E_{y}-f_{m} E_{m}}{f_{2}}
\end{aligned}
$$

\section{Results and Discussion}

The respective effective moduli of composites containing curved fibers and corresponding straight fibers are predicted. Note that these two fibers have the same length. To estimate the effective elastic modulus of composite reinforced by both of wavy CNTs and agglomerated CNTs, the effective elastic stiffness of inclusions is calculated and then figure up the overall property of whole composite system. In this study, the parameters used are presented in Table 1.

Table 1. Parameters and ratios used for numerical calculations.

\begin{tabular}{ccccc}
\hline $\mathrm{E}_{\mathrm{m}}(\mathrm{GPa})$ & $\mathrm{ENT}(\mathrm{GPa})$ & $\mathrm{a} / \lambda$ & $\lambda / \mathrm{d}$ & $v_{\mathrm{m}}=v_{\mathrm{Nt}}$ \\
\hline 2.25 & 450 & 0.05 & 60 & 0.30 \\
\hline
\end{tabular}


The CNTs are divided into concentrated and freely scattered ones in the matrix. The region agglomerated CNTs nearby matrix is assumed to be spherical in shape and the freely scattered ones are thought in a spatial wavy shape as shown in Fig. 1. Then, we replace the spatial wavy shape with a straight fiber and a wafer. So there are three kinds of inclusions in the matrix.

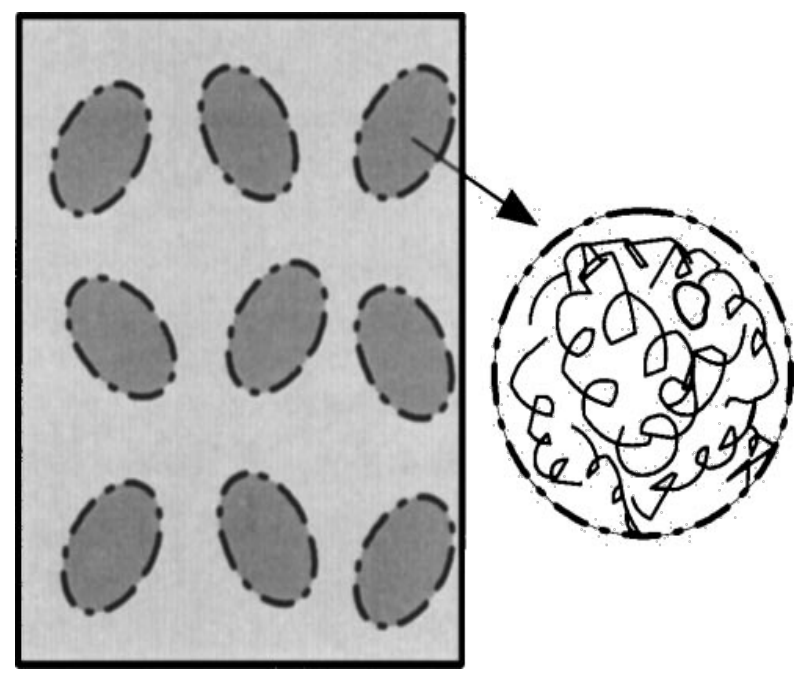

Fig. 1. Inclusion model Proposed for the agglomeration of CNTs.

Fig. 2 gives the variation of effective elastic modulus with the increase of waviness for different volume fractions. It can be found from Fig. 2 that there is almost no obvious influence of waviness on the value of effective elastic modulus when $0<\delta<0.1$. However, the effective elastic moduli are very sensitive to the waviness in the region of $0.1<\delta<0.3$. Moreover, it's noted that the value of effective elastic modulus seams no longer change much when $\delta$ is over 0.3 .

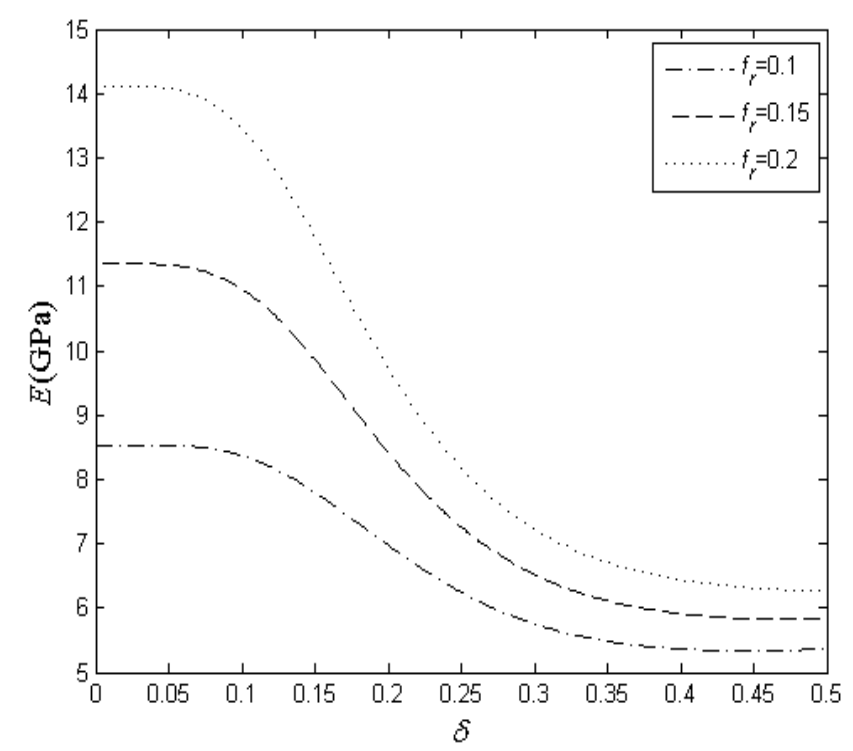

Fig. 2. Effective elastic modulus versus waviness for different volume fractions.

\section{Conclusions}

In this investigation, based on the micromechanics, a new model to replace the wavy carbon nanotubes with effective fibers has been developed. It can be used to predict the effective elastic modulus of nanocomposites with randomly oriented effective fibers. The effects of both waviness 
and agglomeration on the effective elastic modulus are also discussed. As a result, it is found that the stiffness of carbon nanotubes can be reduced, significantly due to the influences of waviness and agglomeration of nanotubes. Moreover, there is a significant influence of waviness and agglomeration on the effective elastic modulus of nanocomposites.

\section{Acknowledgment}

This research was financially supported by the Hebei Natural Science Foundation of China (A2014203139).

\section{References}

[1] H. Hedayati, Aragh B. Sobhani, Influence of graded agglomerated CNTs on vibration of CNT-reinforced annular sectorial plates resting on Pasternak foundation, Applied Mathematics and Computation. 218 (2012) 8715-8735.

[2] Ingo Alig, Petra Pötschke, Dirk Lellinger, Tetyana Skipa, Sven Pegel, Gaurav R. Kasaliwal, Tobias Villmow, Establishment, morphology and properties of carbon nanotube networks in polymer melts, Polymer. 53 (2012) 4-28.

[3] L. Bian, Q. Wang, Influence of the particle size and volume fraction on micro-damage of the composites, Archive of Applied Mechanics. 83(3) (2013) 445-454.

[4] F. Otero, X. Martínez, S. Oller, O. Salomón, Study and prediction of the mechanical performance of a nanotube-reinforced composite, Composite Structures. 94(9) (2012) 2920-2930.

[5] Duc Chinh Pham, Strong-contrast expansion correlation approximations for the effective elastic moduli of multiphase composites, Archive of Applied Mechanics. 82(3) (2012) 377-389.

[6] L. Bian, H. Zhao, Elastic properties of a single-walled carbon nanotube under a thermal environment, Composite Structures. 121 (2015) 337-343.

[7] E. Shady, Y. Gowayed, Effect of nanotube geometry on the elastic properties of nanocomposites, Composite Science and Technology. 70 (2010) 1476-81.

[8] G.I. Giannopoulos, S.K. Georgantzinos, N.K. Anifantis, A semi-continuum finite element approach to evaluate the Young's modulus of single-walled carbon nanotube reinforced composites, Composites Part B. 2010 594-601.

[9] Iman Eslami Afrooz, Andreas chsner, Moones Rahmandoust, Effects of the carbon nanotube distribution on the macroscopic stiffness of composite materials, Computational Materials Science. 51 (2012) 422-9.

[10] C.M. Kuo, K. Takahashi, T.W. Chou, Effect of fiber waviness on the nonlinear elastic behavior of flexible composites, J Compos Mater. 22 (1988) 1004-25. 